\title{
Omnidirectional Reflector based on Transparent Oxide Materials for Light Extraction Enhancement of Organic and Inorganic Solid-state Light Emitting Devices
}

\author{
Kazuyuki Yamae ${ }^{1,2^{*}}$, Hiroshi Fukshima ${ }^{1}$, Tomoya Iwahashi ${ }^{1}$, \\ Masaharu Yasuda $^{1}$, and Kozo Fujimoto ${ }^{2}$ \\ ${ }^{1}$ Panasonic Corporation, Eco Solutions Company, Kadoma, Osaka 571-8686, Japan \\ ${ }^{2}$ Graduated School of Engineering, Osaka University, Suita, Osaka 565-0871, Japan \\ *yamae.kazuyuki@jp.panasonic.com
}

\begin{abstract}
High performance reflector is significant structure for efficiency improvement of organic and inorganic solid-state light emitting devices because a large difference in the refractive index between the light source and air leads to multiple internal reflections at the reflector. Then we focused on wide angular light incidence and designed a total internal omnidirectional reflector (TI-ODR) based on transparent oxide layers. The simulation results showed that light extraction structure with TI-ODR gives $17 \%$ and $4 \%$ efficiency enhancement compared to typical Ag based mirror for organic LED $(n=1.8)$ and inorganic LED $(n=2.4)$, respectively. Then the TI-ODR on blue LED structure was fabricated and that showed enhancement effect of about $8 \%$. The layers are composed of conductive layer for current distribution, low-index thick layer for total internal reflection (TIR) of wide angular light, and high-index layer for the reinforcement of adhesion of layers.
\end{abstract}

Keywords: Solid-state lighting, Light extraction efficiency, Omnidirectional, Oxide material

\section{Introduction}

Solid-state light emitting devices like light emitting diodes (LEDs) and organic light emitting diodes (OLEDs) are widely spreading to the lighting and display industry. Paradigm shift in light sources from the conventional light sources, for example, incandescent lamps and fluorescent tubes, has been accelerating since the action against the environmental issues such as global warming and mercury contamination. Efficiency is a significant specification because the lighting industry occupies approximately $20 \%$ of global energy consumption.

The efficiency of lighting device is usually evaluated by luminous efficacy $[1 \mathrm{~m} / \mathrm{W}$, lumens per watt], which is determined by the product of four factors: luminous efficacy of radiation (LER) $[\mathrm{lm} / \mathrm{W}]$, feeding efficiency (FE) [\%], internal quantum efficiency (IQE) [\%], and light extraction efficiency (LEE) [\%]. LEE has significant impact because the active layer of a solid-state light emitting device typically has high refractive index $(n>1.8)$, and thus, the emitted light is hard to extract into the air because of total internal reflection (TIR) at the light extraction interface. We can get an approximate LEE for isotropic emissive solid-state light sources using Snell's Law. For example, using Equation (1) and assuming emission from cubic media with a flat surface and non-absorbent backside mirror, we find that the LEE is only $10 \%$ for an $\mathrm{InGaN}$ active layer in a blue LED ( $n \sim 2.4)$ device, and $20 \%$ for a green OLED $(n \sim 1.8)$ device. The variable $n$ is the refractive index of the media, and $\theta c$ is the critical angle for light incidence from the media to the air. Low LEE values have driven researchers to use various methods, such as microstructure [1-9] to suppress TIR or highly reflective mirror [10-14] to obtain higher efficiency. 


$$
L E E=\frac{2 \pi I_{0} \int_{0}^{\theta_{c}} \sin \theta d \theta}{2 \pi I_{0} \int_{0}^{\frac{\pi}{2}} \sin \theta d \theta}=1-\cos \theta_{c}=1-\sqrt{1-n^{-2}}
$$

The research group of Panasonic has also reported several methods to enhance the LEE. For example, highly efficient OLED devices by employing the highly refractive substrate like PEN (polyethylene naphthalate) film and the micro lens array structure which is fabricated by highly refractive polymer materials were studied [15-20]. In addition, highly reflective electrode to maximize synergy effect of microstructure was investigated. Total internal reflective omnidirectional reflector (TI-ODR) which is designed to utilize TIR of wide angular incident light by low-index thick layer was developed. The angular integrated reflectance of TI-ODR reached to $99.7 \%$ and indicated LEE enhancement effect of solid-state lighting devices [21]. However, since the multi-layer structure was optimized to maximize optical property, the other properties are insufficient for device and application. In this paper, we further investigated detailed structure of TI-ODR to improve mechanical and electrical property for more practical use in wide applications.

\section{Simulation}

The approach to improve LEE of solid-state lighting device is to maximize $R_{\text {int }}$, angular integrated reflectance defined by Equation (2), of backside mirror. $\theta$ is the incident angle to the reflector. The causal relationship between the microstructure and reflectance of the backside mirror has been already reported with the optical simulation of the LEE [21].

$$
R_{\mathrm{int}}=\frac{\int_{0}^{\pi / 2} R(\theta) \sin \theta d \theta}{\int_{0}^{\pi / 2} \sin \theta d \theta}
$$

The optical simulation for the model of Fig. 1 was conducted with LightTools 8.5, which is a software product of Monte Carlo ray-tracing method manufactured by Synopsys. The model for the solid-state lighting device has dimensions $10 \mathrm{~mm} \times 10 \mathrm{~mm} \times 0.5 \mathrm{~mm}$ and is composed of a micro-structure or flat surface, a mirror edge $\left(R_{\text {int }}\right.$ $=100 \%$ ), a light guide medium which includes the light source, and a backside reflector. The microstructure is chosen from a typical periodic micro lens array (MLA) with pitch fixed at 0.6 $\mathrm{mm}$. The shapes of the MLA can be controlled by setting the aspect ratio, radius, or base angle. The refractive index of the medium is chosen to be $n=2.4$, making this applicable for InGaN blue LED devices. The optical absorbance coefficient of the medium, $\alpha$, is valuable for the range from 0 to $1 \mathrm{~cm}^{-1}$.

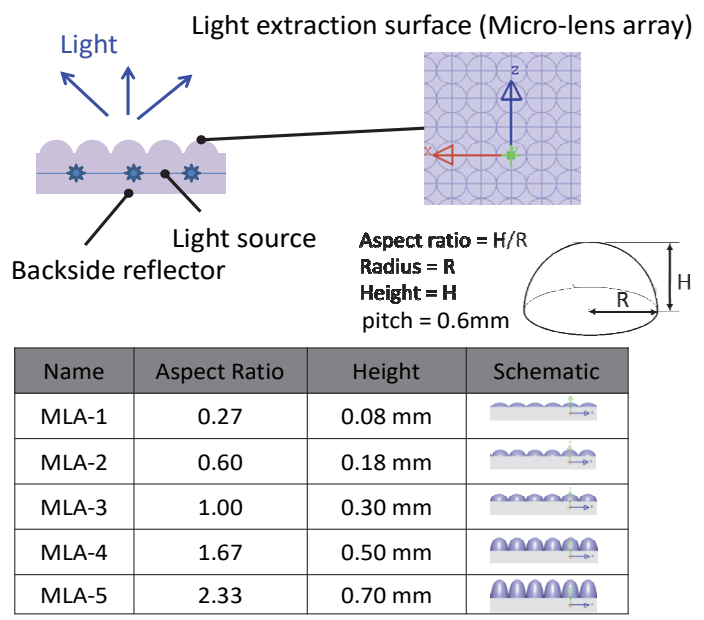

Fig. 1. Simulation model for calculating the light extraction efficiency dependence on reflectance.

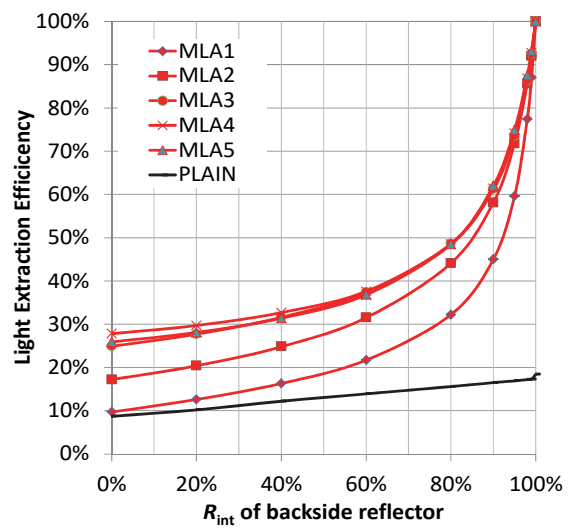

(a)

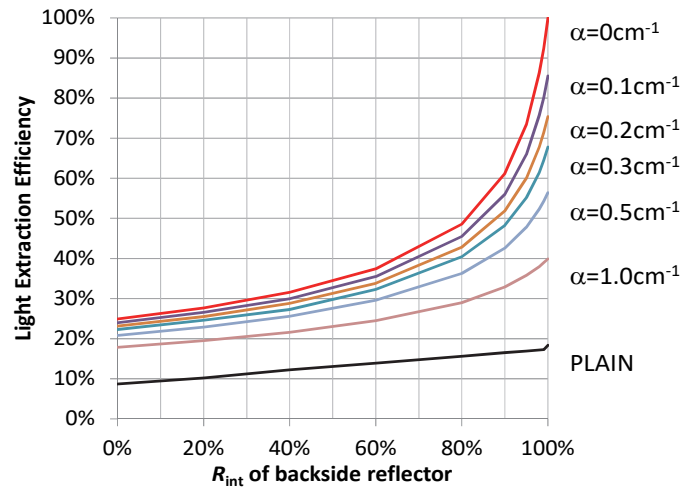

(b)

Fig. 2. Simulated dependence between the LEE and $R_{\text {int }}$ of backside mirror.
(a) LEE for the various micro lens arrays (MLA).
(b) LEE for various absorption coefficients for the case of MLA-3. 
Simulation result is shown in Fig. 2 (a). Particularly in the region where $R_{\text {int }}>95 \%$, this value has significant impact on the light extraction efficiency of solid-state lighting. This result indicates that it should rather focus on improving $R_{\text {int }}$ than the optical design of the microstructure when $R_{\text {int }}$ is close to $100 \%$. This is the principal motivation to develop TI-ODR. However, this trend becomes less important when the media has absorption, as shown in Fig. 2 (b). Then we further examined the case of actual device structure based on the simulation result above.

Figure 3 shows (a) LED and (b) OLED supposing that we introduce TI-ODR to these devices. (a) LED has the transparent conductive layer for current drift since electrical mobility of the contact layer is typically low. In addition, the mirror needs metal pattern to have the function as an electrode. Then the reflectance is controlled by area ratio of dielectric layer. Dielectric layers were carefully chosen from practical oxide materials. In this case, $\mathrm{SiO}_{2}$ and $\mathrm{ZrO}_{2}$ were chosen as low and high index layer, respectively. This is because the pair has been already investigated the capability to apply to optical multi-layer film for the highly reflective mirror [22]. Here, the balance of tensile stress of $\mathrm{SiO}_{2}$ and compressive stress of $\mathrm{ZrO}_{2}$ by the measurement of wafer warpage was examined beforehand. As a result, the best thickness ratio was found that $\mathrm{SiO}_{2}$ and $\mathrm{ZrO}_{2}$ is $1: 5$ to minimize stress and maximize adhesion of TI-ODR in this case [22-24]. Then the thickness ratio was fixed to $1: 5$ in this simulation model. On the other hand, (b) OLED needs low refractive index organic conductive materials as electron transport layer (ETL) to introduce TI-ODR. It is usually hard to realize high conductivity and low refractive index simultaneously in organic materials, so the structure has currently been in study phase yet $[25,26]$. In this simulation model, the lowest refractive index already reported was adopted [25].

The detail of reflector's structure was simulated by ThinFilmView version 2.2.0, which is a Fresnel calculation program for optical thin films manufactured by Nary Software. The spectral characteristics of $n$ and $k$ are applied by using optical properties installed in ThinFilmView except for the materials shown in Fig. 3.

Figure 4 shows the result of the simulation. $R_{\text {int }}$ was improved from $95.0 \%$ to $98.1 \%$ with TI-

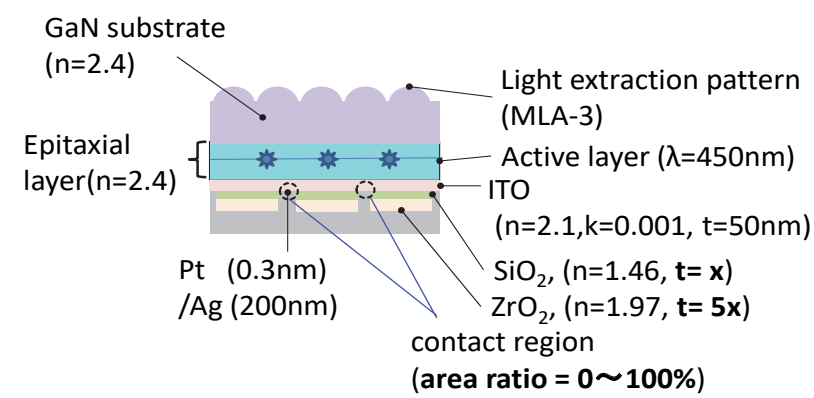

(a) LED

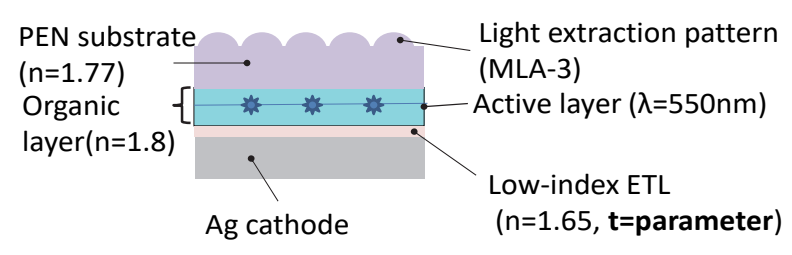

(b) OLED

Fig. 3. Simulation model for calculating the reflectance and light extraction efficiency of devices.

ODR in (a) LED structure. The thickness factor $T$ is critical to the effect because the layer needs the thickness of more than three quarters of optical wavelength in media to demonstrate TIR. Then the enhancement effect is clearly shown in the region of $T>0.5$. This effect leads to the 1.17 of LEE enhancement factor according to the model of MLA-3 in Fig. 1. The enhancement factor is varied by the area ratio of TI-ODR. On the other hand, $R_{\text {int }}$ was improved from $97.9 \%$ to $98.6 \%$ and enhancement factor was 1.04 in (b) OLED structure. The effect of TI-ODR is not enough due to the small refractive index difference between active layer and ETL. In addition, efficiency of OLED is dominated by the multiple factor with the thickness of ETL. For example, the thickness of ETL changes charge balance, cavity effect in organic layer with metal cathode, and influence of surface plasmon at the cathode. These factors directly cause the efficiency of OLED device. Then that should be considered that multiple factors except for the $R_{\text {int }}$ of TI-ODR simultaneously to optimize OLED device structure.

Therefore, we focused on the TI-ODR effect of inorganic LED devices with experiment in this work. 


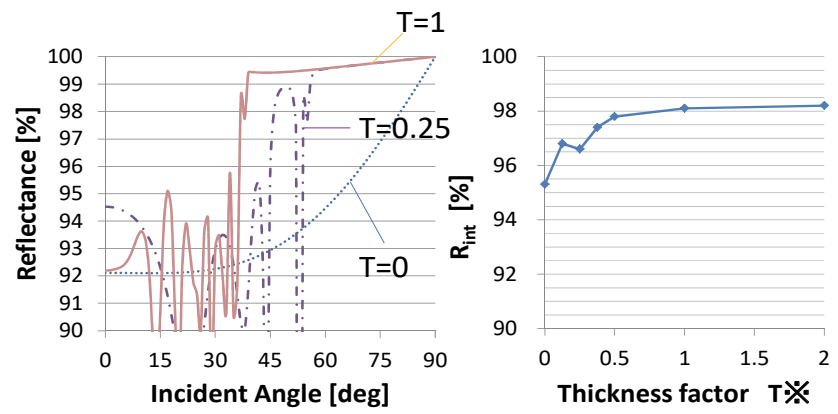

(a) LED

※ Thickness of $\mathrm{SiO}_{2}=450 / 1.46=308 \times \mathrm{T} \quad[\mathrm{nm}]$ $\mathrm{ZrO}_{2}=5 \times 308=1540 \times \mathrm{T}[\mathrm{nm}]$

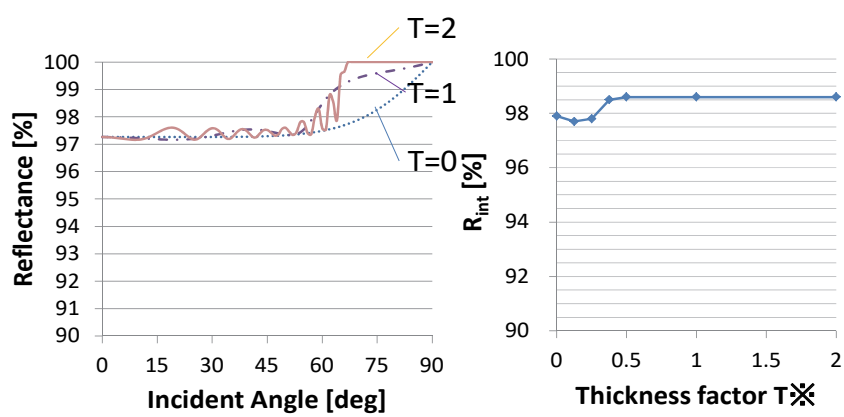

(b) OLED

$※$ Thickness of ETL $=550 / 1.65=333 \times T[n m]$

Fig. 4. Simulated result of the reflectance in the devices.

\section{Experimental method}

The LED device shown in Fig. 3(a) to examine the efficiency enhancement effect by TI-ODR was actually fabricated. First, the textured GaN substrate wafer was prepared. Semiconductor epitaxial layer based on $\mathrm{GaN}$ includes active layer was grown on the substrate by metal organic chemical vapor deposition (MOCVD). Transparent conductive layer (ITO) was fabricated by sputter. The sputter condition was carefully controlled not to give damage to the epitaxial layer. Dielectric layers $\left(\mathrm{SiO}_{2}\right.$ and $\left.\mathrm{ZrO}_{2}\right)$ for TI-ODR was deposited by electron beam (EB). To obtain electrical conduction, the contact metal region was patterned by photolithography. The thickness of these layers was selected in the case of factor $T=0.125$ and 0.5 referred to the simulation result shown in Fig. 4 (a). The area ratio of dielectric layers was settled to $60 \%$ in this case. A basic reference electrode (Pt/Ag) and cover metal $\mathrm{(Au})$ for the bonding was deposited on the dielectric layer pattern. The wafer was diced to obtain LED device chip by mechanical or laser scriber. The LED chip was mounted on the package by typical flip-chip bonding method. We also prepared the reference sample without transparent conductive layer and dielectric layers. The estimated reflectance of each electrode was derived from optical simulation using their refractive index, extinction coefficient, and thickness of layers because the reflectance cannot be obtained directly, especially for the incident angle of larger than the critical angle. The list of the sample is shown in Table 1.

Figure 5 shows the measurement system composed of an integrating sphere and a source meter. The external quantum efficiency (EQE) and driving voltage were measured to evaluate performance of the TI-ODR. The EQE is calculated by input power, total radiant flux, and the photon energy obtained by wavelength. The efficiency enhancement factor was obtained by comparing EQEs.

Table 1. List of the fabricated LED samples.

\begin{tabular}{|c|c|c|c|c|}
\hline sample \# & ITO & Dielectric layers & $\mathrm{Pt} / \mathrm{Ag}$ & Estimated $R_{\text {int }}$ \\
\hline A & $\bigcirc$ & $\begin{array}{c}\mathrm{T}=0.500 \\
\text { area ratio }=60 \%\end{array}$ & $\bigcirc$ & $97.8 \%$ \\
\hline B & $\bigcirc$ & $\begin{array}{c}\mathrm{T}=0.125 \\
\text { area ratio }=60 \%\end{array}$ & $\bigcirc$ & $96.8 \%$ \\
\hline C & - & $\begin{array}{c}\mathrm{T}=0.125 \\
\text { area ratio }=60 \%\end{array}$ & $\bigcirc$ & $97.1 \%$ \\
\hline D & - & - & $\bigcirc$ & $95.0 \%$ \\
\hline
\end{tabular}

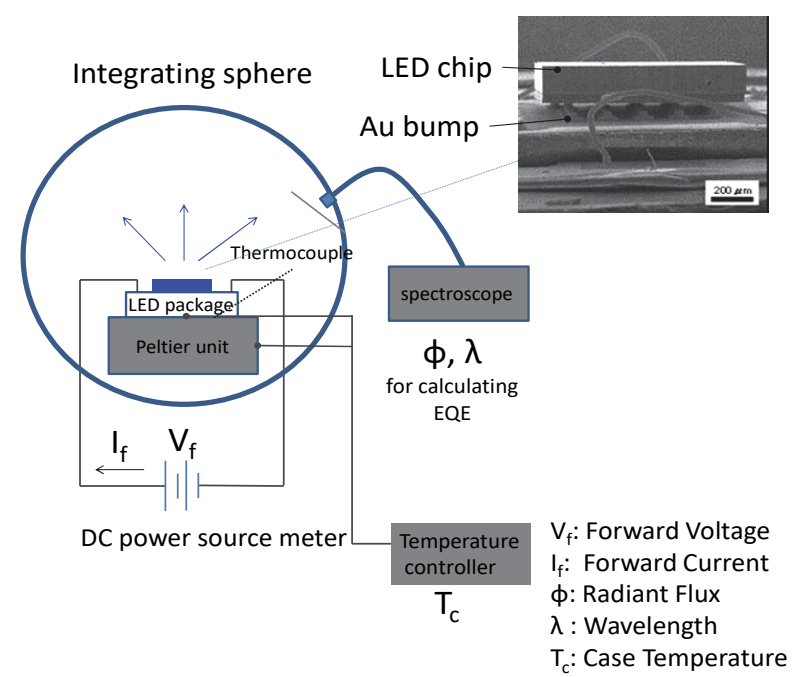

Fig. 5. Measurement system for efficiency enhancement effect of LED with TI-ODR. 


\section{Results and discussion}

Result of measured EQE is shown in Fig. 6. The enhancement factor of $\mathrm{A}, \mathrm{B}, \mathrm{C}$ compared to the reference $\mathrm{D}$ was $1.08,1.06$ and 0.89 , respectively, at a rated current of $350 \mathrm{~mA}$ and a case temperature of 25 degrees Celsius. Maximum enhancement effect of EQE was about $8 \%$.

The EQE of sample A and B was enhanced while the increase of reflectance was slight. The experimental result of the efficiency enhancement factor was similar to the simulated result which is taken into consideration of the area ratio. In addition, significant effect of transparent conductive layer was observed. Without the transparent conductive layer, the $\mathrm{EQE}$ was degraded as the result of sample C. It is assumed that current concentration on the region around conductive layer induces the negative effect of increasing electrical resistance and Joule heating. Then the heat degrades the internal quantum efficiency of the device. The picture shown in Fig. 6 suggests the current concentration. Sample B with ITO layer shows the good uniformity of brightness. On the other hand, sample $\mathrm{C}$ without ITO layer shows the bright spot at the region around conduction bump. As a future work, it should be studied that the higher $R_{\text {int }}$ by TI-ODR with higher area occupation of dielectric layers. Then further optimization of the electrode design is required to achieve better optical and electrical properties simultaneously.
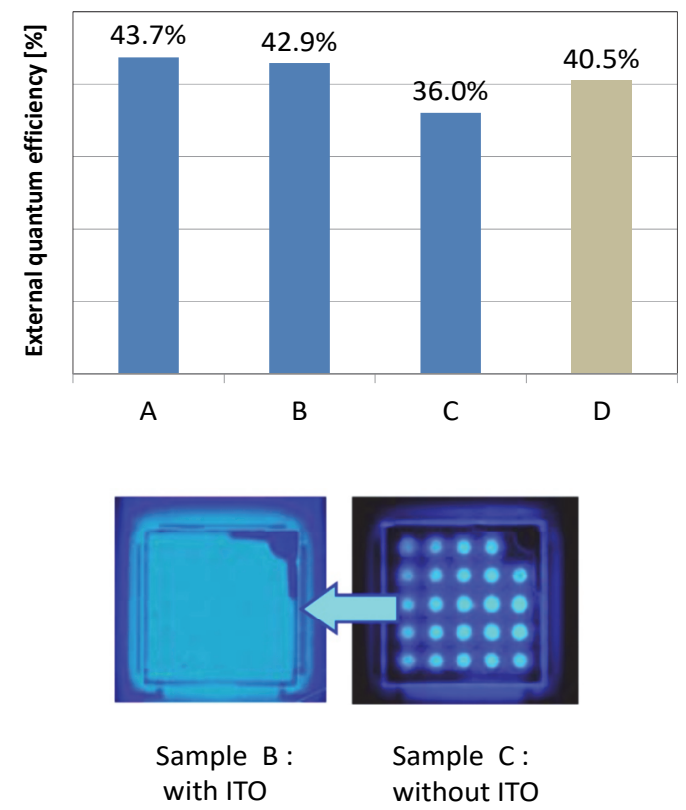

Fig. 6. Experimental result of the efficiency enhance factor of each sample.

\section{Conclusion}

High performance reflector for efficiency improvement of organic and inorganic solid-state light emitting devices to minimize internal loss in the devices was studied. In particular, the TI-ODR based on transparent oxide layers while the mechanical and electrical property is applicable to the devices was carefully designed. The simulation results showed that light extraction structure with TI-ODR gives $17 \%$ and $4 \%$ efficiency enhancement compared to typical $\mathrm{Ag}$ based mirror for inorganic LED $(n=2.4)$ and organic LED $(n=1.8)$, respectively. Then the TI-ODR on the blue LED structure was fabricated and that showed enhancement effect of about $8 \%$.

\section{Acknowledgement}

We acknowledge financial support from Ministry of the Environment in Japan through the project "Cost reduction technical development for the promotion of spreading an energy-saving white LED lighting as technical development business against global warming" for a part of this work.

\section{References}

1. M. Yamada, T. Mitani, Y. Narukawa, S. Shoji, I. Niki, S. Sonobe, K. Deguchi, M. Sano, and T. Mukai, Jpn. J. Appl. Phys., 41 (2002) L1431.

2. H. Wang, Z. Lin, J. Han, L. Zhong, and G. Li, Chin. Phys. B, 24 (2015) 061703.

3. Y. Peng, X. Guo, R. Liang, Y. Mou, H. Cheng, M. Chen, and S. Liu, ACS Photonics, 4 (2017) 2479.

4. P. Mao, F. Sun, H. Yao, J. Chen, B. Zhao, B. Xie, M. Han, and G. Wang, Nanoscale, 6 (2014) 8177.

5. P. Mao, A. Mahapatra, J. Chen, M. Chen, G. Wang, and M. Han, Appl. Mater. Interfaces, 7 (2015) 19179.

6. P. Mao, M. Xu, J. Chen, B. Xie, F. Song, M. Han, and G. Wang, Nanotechnology, 26 (2015) 185201.

7. S. Möller and S. R. Forrest, J. Appl. Phys., 91 (2002) 3324

8. Y. Sun and S. R. Forrest, J. Appl. Phys., 100 (2006) 073106.

9. H. Bae, J. Kim, and C. Hong, Opt. Commun., 415 (2018) 168.

10. T. Gessmann and E. F. Schubert, J. Appl. Phys., 95 (2004) 2203. 
11. J. K. Kim, T. Gessmann, H. Luo, and E. F. Schubert, Appl. Phys. Lett., 84 (2004) 4508.

12. J. K. Kim, J.-Q. Xi and E. F. Shubert, Proc. SPIE, 6134 (2006) 61340D.

13. H. Kim, S.-N. Lee, K.-K. Kim, J. Kwak, and T.-Y. Seong, J. Appl. Phys., 104 (2008) 053111.

14. S. Zhou, B. Cao, S. Yuan, and S. Liu, Appl. Opt., 53 (2014) 8104.

15. K. Yamae, H. Tsuji, V. Kittichungchit, N. Ide, and T. Komoda, J. Soc. Inf. Disp., 21 (2013) 529.

16. K. Yamae, V. Kittichungchit, N. Ide, M. Ota, and T. Komoda, J. Soc. Inf. Disp., 22 (2014) 499.

17. T. Komoda, K. Yamae, V. Kittichungchit, H. Tsuji, and N. Ide, J. Photopolym. Sci. Technol., 25 (2012) 321.

18. H. Tsuji, K. Yamae, V. Kittichungchit, N. Ide, and T. Komoda, J. Photopolym. Sci. Technol., 26 (2013) 415.
19. N. Ide, K. Yamae, V. Kittichungchit, H. Tsuji, M. Ota, and T. Komoda, J. Photopolym. Sci. Technol., 27 (2014) 357.

20. J. Matsuzaki and K. Yamae, J. Photopolym. Sci. Technol., 28 (2015) 329.

21. K. Yamae, H. Fukshima, and K. Fujimoto, Phys. Stat. Sol. A, 215 (2018) 1700775.

22. M. Yasuda, T. Iwahashi, A. Murai, H. Fukshima, K. Yamae, S. F. Chichibu, T. Onuma, and K. Hazu, Japan Patent JP2010-272592A (2010).

23. T. Iwahashi, A. Murai, H. Fukshima, M. Yasuda, and K. Yamae, Japan Patent JP5091823B (2012).

24. H. Fukshima, K. Yamae, M. Yasuda, and T. Iwahashi, Japan Patent JP5186259B (2013).

25. A. Salehi, S. Ho, Y. Chen, C. Peng, H. Yersin, and F. So, Adv. Opt. Mater., 5 (2017) 1700197.

26. D. Yokoyama, K. Nakayama, T. Otani, and J. Kido, Adv. Mater., 24 (2012) 6368. 